\title{
JACOBI DISCRETE APPROXIMATION FOR SOLVING OPTIMAL CONTROL PROBLEMS
}

\author{
MAMDOUH EL-KADY
}

\begin{abstract}
This paper attempts to present a numerical method for solving optimal control problems. The method is based upon constructing the $n$-th degree Jacobi polynomials to approximate the control vector and use differentiation matrix to approximate derivative term in the state system. The system dynamics are then converted into system of algebraic equations and hence the optimal control problem is reduced to constrained optimization problem. Numerical examples illustrate the robustness, accuracy and efficiency of the proposed method.
\end{abstract}

\section{Introduction}

Bellman's dynamic programming and Pontryagin's maximum principle present the most known methods for solving optimal control problems. The computational approaches are considered a very important part of the solution of those problems [19]. In recent years, considerable attention has been given to the use of spectral methods for solving optimal control problems $[2,12,14,15]$.

Part of the difficulty of optimal control is that the first order conditions yield differential equation, which we have to solve to obtain a closed form solution [13]. Hence, to solve optimal control problems we have to study the numerical solutions of differential equations. The same previous attention, the spectral methods play an important role for solving differential equations $[9,17,16]$.

The Jacobi polynomials $P_{n}^{(\alpha, \beta)}(x)$ play important roles in approximation theory and its applications, see $[3,4]$. In his recent work $[7,5,6]$ Doha develops a class of spectral-Galerkin methods for the direct solution of higher order differential equations. One of particular interest here is the Jacobi formula, based on finite Jacobi expansion in terms of power of $x$.

In this paper, a numerical solution for solving optimal control problem is presented. Spectral method is a family of techniques for solving optimal control problems in which the summation in the numerical derivative is accelerated to

Received August 26, 2010.

2010 Mathematics Subject Classification. Primary 65N35, 65L05, 65J10, 49J15.

Key words and phrases. Jacobi polynomials, differentiation and integration matrices, optimal control problem. 
produce a matrix representation that is not only exponentially convergent like the discrete variable representation and other spectral methods but also sparse like traditional finite differences and finite elements [11].

The proposed algorithm describes an alternative technique based on converting the system dynamics, the performance index and the initial or boundary conditions into algebraic equations with unknown coefficients. The differentiation and integration matrices based on Jacobi polynomials are produced here to approximate the system dynamic and performance index, respectively. This approach is based on the expansion of the control and state variables in Jacobi series with unknown coefficients. In the system dynamics, we used collocation method to approximate the derivatives arising in the system dynamics and hence converted to algebraic equations.

In this way, the optimal control problem is converted to Non-Linear Programming (NLP) problem, which consists of minimize the objective function, subject to algebraic constraints. The resulting NLP problem can be solved using well-known solvers such as IPOPT [10] or PPQI [8].

The paper is organized as follows: In Section 2, we introduce definitions of Jacobi polynomial and some of its properties. In Section 3, the differentiation and integration Jacobi matrices are introduced. In Section 4, we describe the approximation of the optimal control problem and the solution technique is summarized. In Section 5, numerical results are given to clarify the proposed method and compared with other methods. In Section 6, remarks and conclusions of the work are presented.

\section{Jacobi polynomials and analytical properties}

In this section some useful notations and results concerning the Jacobi polynomials are introduced. Jacobi polynomials with the real parameter $\alpha, \beta>-1$ are sequence of polynomials $P_{n}^{\alpha, \beta}(x),(n=0,1,2, \ldots)$. Let $N \geq 1$ be an integer, and let $x_{0}, x_{1}, \ldots, x_{N}$ be a set of points in $[-1,1]$, where

$$
-1=x_{0}<x_{1}<\cdots<x_{N-1}<x_{N}=1
$$

are zeros of Jacobi polynomials with ends of interval $[-1,1]$. Consider some of Jacobi polynomials formulae, [1]

$$
\begin{aligned}
& P_{n}^{(\alpha, \beta)}(x)=\sum_{r=0}^{n} \frac{1}{r !(n-r) !} \frac{\Gamma(\alpha+\beta+n+r+1) \Gamma(\alpha+n+1)}{\Gamma(\alpha+\beta+n+1) \Gamma(\alpha+r+1)}\left(\frac{x-1}{2}\right)^{r} \\
& P_{n}^{(\alpha, \beta)}(x)=\sum_{r=0}^{n} \frac{(-1)^{n-r}}{r !(n-r) !} \frac{\Gamma(\alpha+\beta+n+r+1) \Gamma(\beta+n+1)}{\Gamma(\alpha+\beta+n+1) \Gamma(\beta+r+1)}\left(\frac{x+1}{2}\right)^{r} \\
& P_{n}^{(\alpha, \beta)}(x)=\frac{(-1)^{n}}{2^{n} n !}(1-x)^{-\alpha}(1+x)^{-\beta} \frac{d^{n}}{d x^{n}}\left[(1-x)^{\alpha+n}(1+x)^{\beta+n}\right] .
\end{aligned}
$$


An important property of the Jacobi polynomials is that they are orthogonal with respect to the $L^{2}$ inner product on the interval $-1 \leq x \leq 1$ and the weight function is $(1+x)^{\beta}(1-x)^{\alpha}$, i.e.,

$$
\begin{aligned}
\left\langle P_{n}^{(\alpha, \beta)} P_{m}^{(\alpha, \beta)}\right\rangle & =\int_{-1}^{1}(1-x)^{\alpha}(1+x)^{\beta} P_{n}^{(\alpha, \beta)}(x) P_{m}^{(\alpha, \beta)}(x) d x \\
& =\lambda_{n}^{(\alpha, \beta)} \delta_{n m},
\end{aligned}
$$

where

$$
\begin{aligned}
\lambda_{n}^{(\alpha, \beta)} & =\frac{2^{\alpha+\beta+1} \Gamma(\beta+n+1) \Gamma(\alpha+n+1)}{n !(\alpha+\beta+2 n+1) \Gamma(\beta+\alpha+n+1)}, \\
\delta_{n m} & = \begin{cases}0 & \text { if } b \neq m \\
1 & \text { if } n=m .\end{cases}
\end{aligned}
$$

The recurrence relations are giving by:

$$
\begin{aligned}
& 2 n(\alpha+\beta+n)(\alpha+\beta+2 n-2) P_{n}^{(\alpha, \beta)}(x) \\
(5)= & (\alpha+\beta+2 n-1)\left(\alpha^{2}+x(\alpha+\beta+2 n)(\alpha+\beta+2 n-2)\right) P_{n-1}^{(\alpha, \beta)}(x) \\
& -2(\alpha+n-1)(\beta+n-1)(\alpha+\beta+2 n) P_{n-2}^{(\alpha, \beta)}(x)
\end{aligned}
$$

with $P_{0}^{(\alpha, \beta)}(x)=1$ and $P_{1}^{(\alpha, \beta)}(x)=(\alpha+1)+(\alpha+\beta+2)\left(\frac{x-1}{2}\right)$.

Important special cases of Jacobi polynomials are the Chebyshev polynomials of first and second kinds and Legendre polynomials (these are also instances of Ultraspherical polynomials) which are directly obtainable from Jacobi polynomials with specified parameters as follows:

$T_{j}(x)$ are the Chebyshev polynomials of the first kind $\left(\alpha=\beta=-\frac{1}{2}\right)$,

$$
P_{j}^{\left(-\frac{1}{2}, \frac{1}{2}\right)}(x)=\frac{1 \cdot 3 \cdots(2 j-1)}{2 \cdot 4 \cdots 2 j} T_{j}(x) .
$$

$U_{j}(x)$ are the Chebyshev polynomials of the second kind $\left(\alpha=\beta=\frac{1}{2}\right)$,

$$
U_{j}^{\left(\frac{1}{2}, \frac{1}{2}\right)}(x)=2 \frac{1 \cdot 3 \cdots(2 j+1)}{2 \cdot 4 \cdots(2 j+2)} U_{j}(x) .
$$

$L_{j}(x)$ are the Legendre polynomials $(\alpha=\beta=0)$,

$$
P_{j}^{(0,0)}(x)=L_{j}(x) .
$$

Finally, $C_{n}^{(\alpha)}(x)$ are the Ultraspherical polynomials $(\alpha=\beta)$,

$$
C_{n}^{(\alpha)}(x)=\frac{n ! \Gamma(\alpha+1 / 2)}{\Gamma(n+\alpha+1 / 2)} P_{n}^{(\alpha-1 / 2, \alpha-1 / 2)}(x) .
$$

The higher derivatives of the Jacobi polynomials are given by the following theorem. 
Theorem 1. The $n$-th derivatives of the Jacobi polynomials are given by

$$
\frac{d^{n}}{d x^{n}} P_{n}^{(\alpha, \beta)}(x)=\sum_{r=0}^{n} \sum_{k=0}^{n} H_{n, r}^{(\alpha, \beta)}(x+1)^{n-r-k}(x-1)^{r-n+k},
$$

where

$$
H_{n, r}^{(\alpha, \beta)}=\frac{n !}{r ! k !(n-k) !(n-r) !} \frac{\Gamma(\beta+n+1) \Gamma(\alpha+n+1) \Gamma(n-r+1) \Gamma(r+1)}{\Gamma(\beta+n-r+1) \Gamma(\alpha+r+1) \Gamma(n-r-k+1) \Gamma(r-n+k+1)} .
$$

Proof. From Rodrigue's formula of the Jacobi polynomials

$$
P_{n}^{(\alpha, \beta)}(x)=\frac{(-1)^{n}}{2^{n} n !}(1-x)^{-\alpha}(1+x)^{-\beta} \frac{d^{n}}{d x^{n}}\left[(1-x)^{\alpha+n}(1+x)^{\beta+n}\right] .
$$

Leiboniz's theorem for the $n$-th derivative of a product gives

$$
\begin{aligned}
& \frac{d^{n}}{d x^{n}}\left\{(1-x)^{\alpha+n}(1+x)^{\beta+n}\right\} \\
= & \sum_{r=0}^{n} \frac{n !}{r !(n-r) !}\left\{\frac{d^{r}}{d x^{r}}(1+x)^{\beta+n}\right\}\left\{\frac{d^{n-r}}{d x^{n-r}}(1-x)^{\alpha+n}\right\} \\
= & \sum_{r=0}^{n} \frac{n !}{r !(n-r) !}(-1)^{n-r} \frac{\Gamma(\beta+n+1) \Gamma(\alpha+n+1)}{\Gamma(\beta+n-r+1) \Gamma(\alpha+r+1)}(1+x)^{\beta+n-r}(1-x)^{\alpha+r} .
\end{aligned}
$$

Hence,

$$
P_{n}^{(\alpha, \beta)}(x)=\sum_{r=0}^{n} \phi_{n, r}^{(\alpha, \beta)}(x+1)^{n-r}(x-1)^{r}
$$

where

$$
\phi_{n, r}^{(\alpha, \beta)}=\frac{(-1)^{2 n-r}}{r !(n-r) !} \frac{\Gamma(\beta+n+1) \Gamma(\alpha+n+1)}{2^{n} \Gamma(\beta+n-r+1) \Gamma(\alpha+r+1)} .
$$

The $n$-th derivatives of $P_{n}^{(\alpha, \beta)}(x)$ with respect to $x$ are:

$$
\begin{gathered}
\frac{d^{n}}{d x^{n}} P_{n}^{(\alpha, \beta)}(x)=\frac{d^{n}}{d x^{n}} \sum_{r=0}^{n} \phi_{n, r}^{(\alpha, \beta)}\left\{(x+1)^{n-r}(x-1)^{r}\right\}, \\
\frac{d^{n}}{d x^{n}} P_{n}^{(\alpha, \beta)}(x)=\sum_{r=0}^{n} \sum_{k=0}^{n} H_{n, r}^{(\alpha, \beta)}(x+1)^{n-r-k}(x-1)^{r-n+k},
\end{gathered}
$$

where

$$
H_{n, r}^{(\alpha, \beta)}=\frac{n !}{r ! k !(n-k) !(n-r) !} \frac{\Gamma(\beta+n+1) \Gamma(\alpha+n+1) \Gamma(n-r+1) \Gamma(r+1)}{2^{n} \Gamma(\beta+n-r+1) \Gamma(\alpha+r+1) \Gamma(n-r-k+1) \Gamma(r-n+k+1)} .
$$

For the Jacobi polynomials, the following properties are considered in this work:

$$
\begin{aligned}
P_{n}^{(\alpha, \beta)}(-x) & =(-1)^{n} P_{n}^{(\beta, \alpha)}(x), \\
P_{n}^{(\alpha, \beta)}(1) & =\frac{\Gamma(\alpha+n+1)}{n ! \Gamma(\alpha+1)},
\end{aligned}
$$




$$
P_{n}^{(\alpha, \beta-1)}(x)-P_{n}^{(\alpha-1, \beta)}(x)=P_{n-1}^{(\alpha, \beta)}(x) .
$$

Theorem 2. The integration of Jacobi polynomials is expressed in terms of power of $x_{i}$ as follows:

$$
\int_{-1}^{x_{i}} P_{n}^{(\alpha, \beta)}(x) d x=\sum_{r=0}^{n} K_{n, r}^{(\alpha, \beta)} \frac{1}{(r+1)}\left[\left(x_{i}-1\right)^{r+1}-(-2)^{r+1}\right],
$$

where

$$
K_{n, r}^{(\alpha, \beta)}=\frac{1}{2^{r} r !(n-r) !} \frac{\Gamma(\alpha+\beta+n+r+1) \Gamma(\alpha+n+1)}{\Gamma(\alpha+\beta+n+1) \Gamma(\alpha+r+1)} .
$$

Proof. From the equation (1), we have

$$
\begin{aligned}
& \int_{-1}^{x_{i}} P_{n}^{(\alpha, \beta)}(x) d x \\
= & \sum_{r=0}^{n} \frac{1}{2^{r} r !(n-r) !} \frac{\Gamma(\alpha+\beta+n+r+1) \Gamma(\alpha+n+1)}{\Gamma(\alpha+\beta+n+1) \Gamma(\alpha+r+1)} \int_{-1}^{x_{i}}(x-1)^{r} d x \\
= & \sum_{r=0}^{n} K_{n, r}^{(\alpha, \beta)} \frac{1}{(r+1)}\left[\left(x_{i}-1\right)^{r+1}-(-2)^{r+1}\right]
\end{aligned}
$$

where

$$
K_{n, r}^{(\alpha, \beta)}=\frac{1}{2^{r} r !(n-r) !} \frac{\Gamma(\alpha+\beta+n+r+1) \Gamma(\alpha+n+1)}{\Gamma(\alpha+\beta+n+1) \Gamma(\alpha+r+1)} .
$$

\section{Jacobi pseudospectral approximations}

Here, we present Jacobi pseudospectral approximations of a function $f(x) \in$ $C^{\infty}[-1,1]$, at the set of $(N+1)$ points, these points are zeros of $\left(1-x^{2}\right) P_{N-1}^{(\alpha, \beta)}(x)$, i.e.

$$
\left\{x_{i}:\left(1-x^{2}\right) P_{N-1}^{(\alpha, \beta)}(x)=0, i=0,1, \ldots, N\right\},
$$

where $N$ is the degree of the approximation.

Theorem 3. Consider the following discrete approximation of $f(x)$ by using Jacobi polynomials, i.e.,

$$
f(x)=\sum_{j=0}^{N} a_{j} P_{j}^{(\alpha, \beta)}(x) .
$$

Then the coefficients $a_{j}$ are given by:

$$
a_{j}=\frac{1}{\lambda_{j}^{(\alpha, \beta)}} \sum_{k=0}^{N} A_{k}^{(\alpha, \beta)} P_{j}^{(\alpha, \beta)}\left(x_{k}\right) f\left(x_{k}\right), j=0, \ldots, N,
$$

where

$$
A_{k}^{(\alpha, \beta)}=\frac{2^{\alpha+\beta+1} \Gamma(\alpha+N+1) \Gamma(\beta+N+1)}{\Gamma(N+1) \Gamma(\alpha+\beta+N+1)\left(1-x_{k}^{2}\right)\left(P_{N}^{\prime(\alpha, \beta)}\left(x_{k}\right)\right)^{2}}
$$


and $\lambda_{j}^{(\alpha, \beta)}$ are given in the equation (4).

Proof. By using Gauss quadrature formulae for distinct zeros points of Jacobi polynomials:

$$
-1<x_{N-1} \leq \cdots \leq x_{1}<1,
$$

and the weight function $w(x)=(1-x)^{\alpha}(1+x)^{\beta}$ on the interval $(-1,1)$, we have the constants $a_{1}, \ldots, a_{N-1}$ such that:

$$
a_{j}=\frac{1}{\lambda_{j}^{(\alpha, \beta)}} \sum_{k=1}^{N-1} A_{k}^{(\alpha, \beta)} P_{j}^{(\alpha, \beta)}\left(x_{k}\right) f\left(x_{k}\right), \quad j=1, \ldots, N-1,
$$

where

$$
A_{k}^{(\alpha, \beta)}=\frac{2^{\alpha+\beta+1} \Gamma(\alpha+N+1) \Gamma(\beta+N+1)}{\Gamma(N+1) \Gamma(\alpha+\beta+N+1)\left(1-x_{k}^{2}\right)\left(P_{N}^{\prime(\alpha, \beta)}\left(x_{k}\right)\right)^{2}},
$$

$P_{N}^{\prime(\alpha, \beta)}(x)$ is the first derivative of Jacobi polynomials given by (6) with $n=1$. The values of $a_{0}$ and $a_{n}$ can be determined by using properties in Section 2, as follows:

$$
\begin{aligned}
f(-1) & =a_{0} P_{0}^{(\alpha, \beta)}(-1)+a_{N} P_{N}^{(\alpha, \beta)}(-1)+\sum_{j=1}^{N-1} a_{j} P_{j}^{(\alpha, \beta)}(-1), \\
f(1) & =a_{0} P_{0}^{(\alpha, \beta)}(1)+a_{N} P_{N}^{(\alpha, \beta)}(1)+\sum_{j=1}^{N-1} a_{j} P_{j}^{(\alpha, \beta)}(1) .
\end{aligned}
$$

\subsection{Jacobi pseudospectral integration matrix}

The integration of a function $f(x)$ is approximated by interpolating the function with Jacobi polynomial at set of $(N+1)$ point. That is,

$$
\begin{aligned}
\left(I_{N} f\right)= & a_{0} P_{0}^{(\alpha, \beta)}(x)+a_{N} P_{N}^{(\alpha, \beta)}(x) \\
& +\sum_{j=1}^{N-1}\left\{\frac{1}{\lambda_{j}^{(\alpha, \beta)}} \sum_{k=1}^{N-1} A_{k}^{(\alpha, \beta)} P_{j}^{(\alpha, \beta)}\left(x_{k}\right) f\left(x_{k}\right)\right\} P_{j}^{(\alpha, \beta)}(x) \\
= & a_{0} P_{0}^{(\alpha, \beta)}(x)+a_{N} P_{N}^{(\alpha, \beta)}(x) \\
& +\sum_{k=1}^{N-1}\left\{A_{k}^{(\alpha, \beta)} \sum_{j=1}^{N-1} \frac{1}{\lambda_{j}^{(\alpha, \beta)}} P_{j}^{(\alpha, \beta)}\left(x_{k}\right) P_{j}^{(\alpha, \beta)}(x)\right\} f\left(x_{k}\right) .
\end{aligned}
$$

The integration of $f(x)$ can be approximated as follows:

$$
\begin{aligned}
& \int_{-1}^{x_{i}} f(x) d x \\
= & a_{0} \int_{-1}^{x_{i}} P_{0}^{(\alpha, \beta)}(x) d x+a_{N} \int_{-1}^{x_{i}} P_{N}^{(\alpha, \beta)}(x) d x
\end{aligned}
$$




$$
\sum_{j=1}^{N-1}\left\{A_{k}^{(\alpha, \beta)} \sum_{j=1}^{N-1} \frac{1}{\lambda_{j}^{(\alpha, \beta)}} P_{j}^{(\alpha, \beta)}\left(x_{k}\right) \int_{-1}^{x_{i}} P_{j}^{(\alpha, \beta)}(x) d x\right\} f\left(x_{k}\right), i=0, \ldots, N
$$

using Theorem 2, we get:

$$
\begin{aligned}
\int_{-1}^{x_{i}} f(x) d x= & a_{0}\left(x_{i}+1\right)+a_{N} \sum_{r=0}^{N} K_{N, r}^{(\alpha, \beta)} \frac{1}{(r+1)}\left[\left(x_{i}-1\right)^{r+1}-(-2)^{r+1}\right] \\
& +\sum_{k=1}^{N-1} b_{i, k} f\left(x_{k}\right) .
\end{aligned}
$$

The matrix form is:

$$
\left[\int_{-1}^{x_{i}} f(x) d x\right]=\mathbf{A}+\mathbf{C}+\mathbf{B}[f]
$$

where the vectors $\mathbf{A}$ and $\mathbf{C}$ are given by the elements $\mathbf{A}_{i}=\left\{a_{0}\left(x_{i}+1\right)\right\}$, $i=0, \ldots, N$,

and

$$
\mathbf{A}=\left[\begin{array}{lll}
A_{0} & \cdots & A_{N}
\end{array}\right]^{T}
$$

$$
\mathbf{C}_{i}=\left\{a_{N} \sum_{r=0}^{N} K_{N, r}^{(\alpha, \beta)} \frac{\left[\left(x_{i}-1\right)^{r+1}-(-2)^{r+1}\right]}{(r+1)}\right\}
$$

for $i=0, \ldots, N$, are the elements of the vector:

$$
\mathbf{C}_{i}=\left[\begin{array}{lll}
C_{0} & \cdots & C_{N}
\end{array}\right]^{T} .
$$

Finally, the matrix:

$$
\mathbf{B}=\left(\begin{array}{ccc}
b_{0,1} & \cdots & b_{0, N-1} \\
\vdots & & \vdots \\
b_{N, 1} & \cdots & b_{N, N-1}
\end{array}\right)
$$

have the elements:

(14) $b_{i, k}=\left\{A_{k}^{(\alpha, \beta)} \sum_{j=1}^{N-1} \sum_{r=0}^{N} \frac{K_{j, r}^{(\alpha, \beta)}}{(r+1) \lambda_{j}^{(\alpha, \beta)}} P_{j}^{(\alpha, \beta)}\left(x_{k}\right)\left[\left(x_{i}-1\right)^{r+1}-(-2)^{r+1}\right]\right\}$ for $i=0, \ldots, N$, and $k=1, \ldots, N-1$.

The elements of the column matrix $[f]$ are given by $f\left(x_{k}\right) ; k=0, \ldots, N$ with the first and last elements are $f\left(x_{0}\right)=f(-1), f\left(x_{N}\right)=f(1)$.

\subsection{Jacobi pseudospectral differentiation matrix}

In this section, the $n$-th approximate derivatives of $f(x)$ are constructed by using Jacobi interpolation polynomials (12) and the equation (6) as following:

$$
f^{(n)}\left(x_{i}\right)=\sum_{k=0}^{N} d_{i, k}^{(n)} f\left(x_{k}\right), i=0,1, \ldots, N
$$


where

$$
d_{i, k}^{(n)}=A_{k}^{(\alpha, \beta)} \sum_{j=0}^{N} \sum_{r=0}^{N} \sum_{s=0}^{n} \frac{H_{N, n}^{(\alpha, \beta)}}{\lambda_{j}^{(\alpha, \beta)}} P_{j}^{(\alpha, \beta)}\left(x_{k}\right)\left(x_{i}+1\right)^{N-r-s}\left(x_{i}-1\right)^{r-n+s} .
$$

This can be written in matrix form as follows:

$$
\left[\frac{d^{n}}{d x^{n}} f\right]=D^{(n)}[f] \text {. }
$$

Where $D^{(n)}$ is known $(N+1) \times(N+1)$ matrix and their elements given by the equation (15). This formula gives us an improvement of the results as shown in the numerical examples.

\section{Optimal control problem formulation}

In modern control systems design it is sometimes necessary to design controllers that not only effectively control the behavior of a system, but also minimize or maximize some user defined criteria such as energy or time conservation, or time constraints imposed by the environment. Optimal control theory provides the mathematical tools for solving problems like these, either analytically or through computer iterative methods, by formulating the user criteria into a cost function and using the state equation representation for the system dynamics [19].

The general formulation of the optimal control problem is as follows: Let the system states be represented by an $n$-dimensional vector, where $n$ is the order of the system. Let the control variables (input) be represented by an $m$-dimensional vector $u$. The system can (formally called system dynamic) be represented by a set of differential equations of the form:

$$
\begin{gathered}
\frac{d x(\tau)}{d \tau}=F(x(\tau), u(\tau), \tau) \\
x\left(\tau_{0}\right)=x_{0}
\end{gathered}
$$

where $F$ are general expressions of $x(\tau)$ and $u(\tau), \tau_{0}$ is the initial time and $x_{0}$ is an $n$-dimensional set of initial conditions. The objective is to find a control input $u(\tau)$ that will drive the system from the initial point $x_{0}$ in the state space to the final point $\tau_{f}$, and at the same time minimize a cost functional $J$ (formally called performance index) given by:

$$
J=h\left(x\left(\tau_{f}\right), \tau_{f}\right)+\int_{\tau_{0}}^{\tau_{f}} g(x(\tau), u(\tau), \tau) d \tau,
$$

where $\tau_{f}$ represents the end of the control interval, $h$ and $g$ are user defined penalty expressions. Consider the following transformation:

$$
t=\frac{2 \tau-\left(\tau_{f}+\tau_{0}\right)}{\left(\tau_{f}-\tau_{0}\right)}
$$


is introduced in order to use Jacobi polynomials defined on the interval $[-1,1]$. It follows that equations (17)-(19) are replaced by:

$$
\begin{gathered}
\frac{d x(t)}{d t}=F(x(t), u(t), t),-1 \leq t \leq 1, \\
x(-1)=x_{0}, \\
J=h(x(1))+\int_{-1}^{1} g(x(t), u(t), t) d t .
\end{gathered}
$$

The numerical algorithm is based on applying pseudospectral Jacobi method to approximate the derivatives and integral appear in the system dynamics and objective functional, respectively, to convert the optimal control problem into non-linear programming problem. To this end, the control variables are approximated by a finite series of Jacobi polynomial as follows: Consider the differentiation and integration matrices $D$ and $B$ pseudospectral Jacobi approximation, respectively. Then we have the following approximations:

$$
\begin{gathered}
x^{(n)}\left(t_{i}\right)=\sum_{j=0}^{N} d_{i, j}^{(n)} x\left(t_{i}\right), \quad i=0,1, \ldots, N, \\
\int_{-1}^{1} x(t) d t=\sum_{j=0}^{N} b_{N, j} x\left(t_{j}\right),
\end{gathered}
$$

where $\left(b_{i, j}\right)$ are the elements of the matrix $B$ given in the equation $(13),\left(d_{i, j}\right)$ are the elements of the matrix $D$ given in the equation (16). By expanding the control variable in Jacobi series of order, we have:

$$
u_{m}(t)=\sum_{k=0}^{m} a_{k} P_{k}^{(\alpha, \beta)}(t),
$$

where $P_{k}^{(\alpha, \beta)}(t)$ are the $k$-th Jacobi polynomials.

The system dynamics (20) can be approximated as follows:

$$
\sum_{j=0}^{N} d_{i j} x\left(t_{j}\right)=F\left(x\left(t_{i}\right), \sum_{k=0}^{m} a_{k} P_{k}^{(\alpha, \beta)}(t), t_{j}\right),
$$

which can be written in the form:

$$
g[\gamma, \lambda]=0
$$

where

$$
\gamma=\left[x\left(t_{0}\right), x\left(t_{1}\right), \ldots, x\left(t_{N}\right)\right], \lambda=\left[a_{0}, a_{1}, \ldots, a_{m}\right] .
$$

The performance index (22) can be approximated as follows:

$$
J=h\left(x_{N}\right)+\sum_{j=1}^{N-1} b_{N, j} g\left(x\left(t_{j}\right), u\left(t_{j}\right), t_{j}\right),
$$




$$
J=J(\gamma, \lambda) .
$$

Generally, $J$ is nonlinear in $\gamma, \lambda$.

We have confined attention in the previous paragraph to equality constraints. In fact, any inequality constraint can be converted to an equality constraint by introducing an extra 'slack' variable.

The inequality constraint $g_{k}(x)<0$ can be converted to equality constraint by introducing the slack variable $x_{N+k}$ to be

$$
g_{k}(x)+x_{N+k}^{2}=0,
$$

and the inequality constraint $g_{k}(x)>0$ can be converted to equality constraint by introducing the slack variable $x_{N+k}$ to be

$$
g_{k}(x)-x_{N+k}^{2}=0 .
$$

The optimal control problem has been reduced to a parameter optimization problem, i.e.,

$$
\begin{array}{ll}
\text { Minimize } & J=J(\gamma, \lambda) . \\
\text { Subject to } & g[\gamma, \lambda]=0 .
\end{array}
$$

Any techniques are available in such case such as Lagrange multiplier or penalty approaches. We prefer the penalty function approach with partial quadratic interpolation method (PPQI) as [8]. Urabe [18] has described a method to determine very accurately numerical solution of nonlinear ordinary differential equations, and has shows how to study the existence and uniqueness problem of an exact solution near the calculated Chebyshev approximation, and how estimate the error on the approximation, i.e.,

$$
\left|J\left(\gamma_{N+1}, \lambda_{N+1}\right)-J\left(\gamma_{N}, \lambda_{N}\right)\right|<\varepsilon .
$$

\section{Numerical results}

In this section, we present some numerical results using the proposed method and compare the results with other works. The performance of the proposed Jacobi approach was evaluated by identifying parameters for number of variables in the system dynamic.

Example 1. Find the control $u(t)$ that

$$
\begin{array}{ll}
\text { Minimizes } & J=\frac{1}{2} \int_{-1}^{1}\left[x_{1}^{2}(t)+x_{2}^{2}(t)+0.005 u^{2}(t)\right] d t . \\
\text { Subject to } & 2 x_{1}^{\prime}(t)=x_{2}(t), \quad 2 x_{2}^{\prime}(t)=-x_{2}(t)+u(t),
\end{array}
$$

and

$$
x_{1}(-1)=0, \quad x_{2}(-1)=-1 .
$$


Substituting from equations (23) and (25) into the objective and system dynamics (30)-(32), we have the following constrained optimization problem

Minimize $\quad J=\frac{1}{2} \sum_{j=1}^{N-1} b_{N, j}\left[x_{1}^{2}\left(t_{j}\right)+x_{2}^{2}\left(t_{j}\right)+0.005\left(\sum_{k=0}^{m} a_{k} P_{k}^{(\alpha, \beta)}\left(t_{j}\right)\right)^{2}\right]$.

Subject to $\quad G_{1 i}=: 2 \sum_{j=0}^{N} d_{i, j} x_{1}\left(t_{j}\right)-x_{2}\left(t_{i}\right)=0, i=0, \ldots, N$,

$$
G_{2 i}=: 2 \sum_{j=0}^{N} d_{i, j} x_{2}\left(t_{j}\right)+x_{2}\left(t_{i}\right)-\sum_{k=0}^{m} a_{k} P_{k}^{(\alpha, \beta)}\left(t_{i}\right)=0, i=0, \ldots, N,
$$

and

$$
x_{1}(-1)=0, \quad x_{2}(-1)=-1 .
$$

This parameter optimization problem can be solved by using PPQI. In case of $N=8$ and $M=8$ with $(\alpha, \beta)=(0.99,0.26)$ we got the optimal objective functional $J^{*}=0.0693617$. A comparison between the present method and other methods are given in Table 1.

Table 1: An objectives of Example 1

\begin{tabular}{|c|c|}
\hline Methods & $J^{*}$ \\
\hline H. Jaddu [11] & 0.0693689 \\
\hline Present method & 0.0693617 \\
\hline Exact solution & 0.0693609 \\
\hline
\end{tabular}

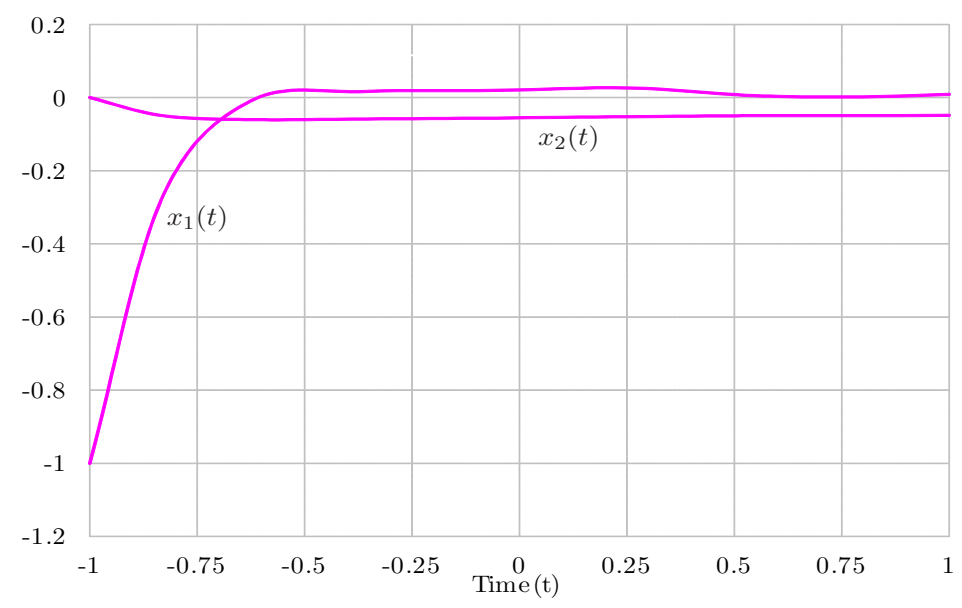

Figure 1. States $x_{1}(t), x_{2}(t)$ of Example 1 


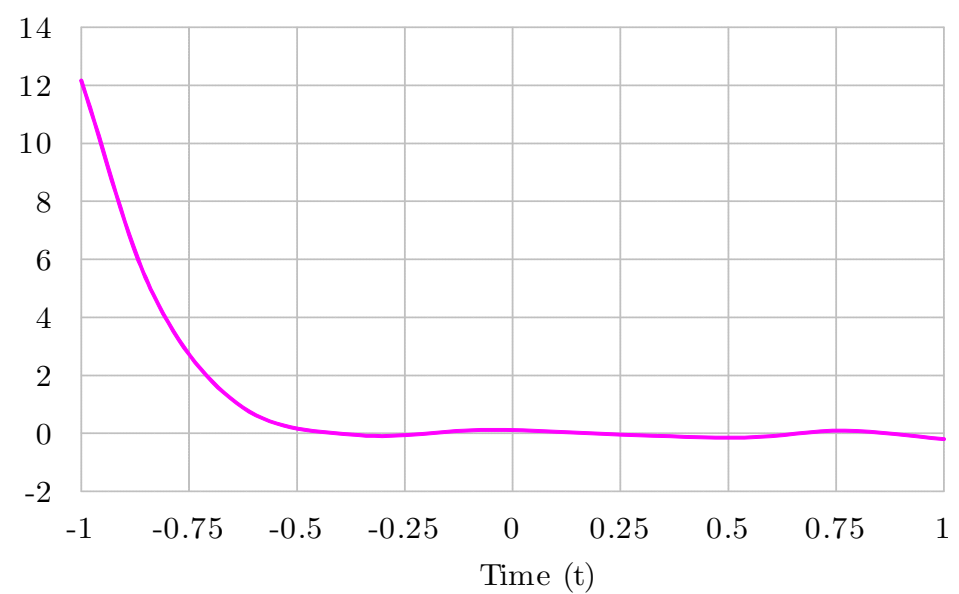

Figure 2. Control variable $u(t)$ of Example 1

Example 2. Solve Example 1 with auxiliary inequality constraint:

$$
x_{2}(t)-2 t^{2}+0.5 \leq 0 .
$$

The present method was applied as above with the following additional equality constraints:

$$
G_{3 i}=x_{2}\left(t_{i}\right)-2 t_{i}^{2}+0.5+h_{i}^{2}=0, \quad i=0, \ldots, N .
$$

In case of $N=8, M=8$ and $(\alpha, \beta)=(0.99,0.60)$, the optimal objective functional was $J^{*}=0.16917833$. Comparisons between the present method and other methods are given in Table 2.

Table 2: An objectives of Example 2

\begin{tabular}{|c|c|}
\hline Methods & $J^{*}$ \\
\hline Vlassenbroeck [19] & 0.18000000 \\
\hline H. Jadu [11] & 0.1708488 \\
\hline Present method & 0.16917833 \\
\hline
\end{tabular}

The simulation graphs for the problem are given in Figures 1, 3 which consists of the states variable $x_{1}, x_{2}$, these variables satisfying the original system (31) and initial condition (32). The controlled variable $u$ corresponding to states variables of the criterion problem are described in Figures 2, 4.

\section{Conclusion}

In this paper a Jacobi expansion method has been used to convert the optimal control problem into a parameter constrained optimization problem. The Penalty partial quadratic interpolation (PPQI) technique has been used to 


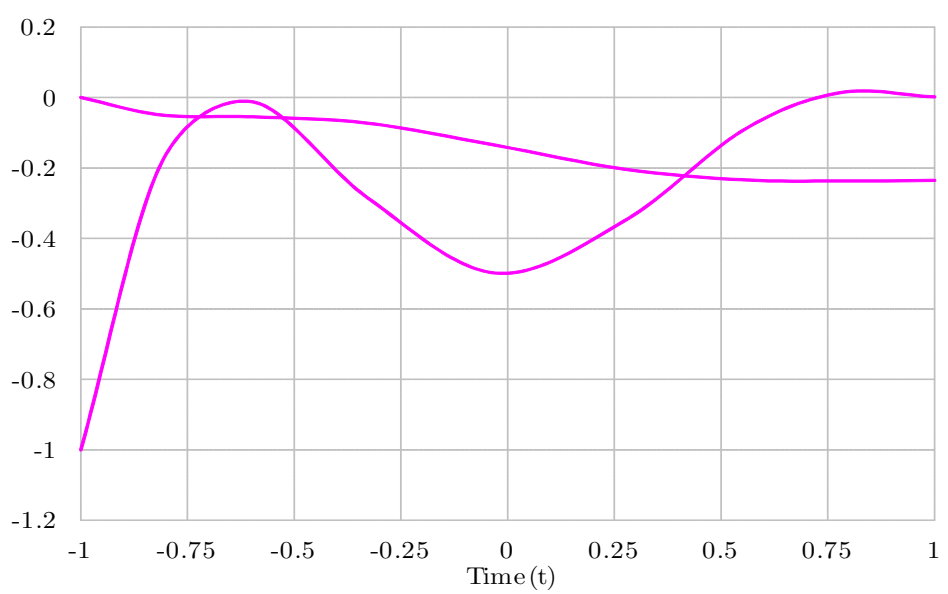

Figure 3. States $x_{1}(t), x_{2}(t)$ of Example 2

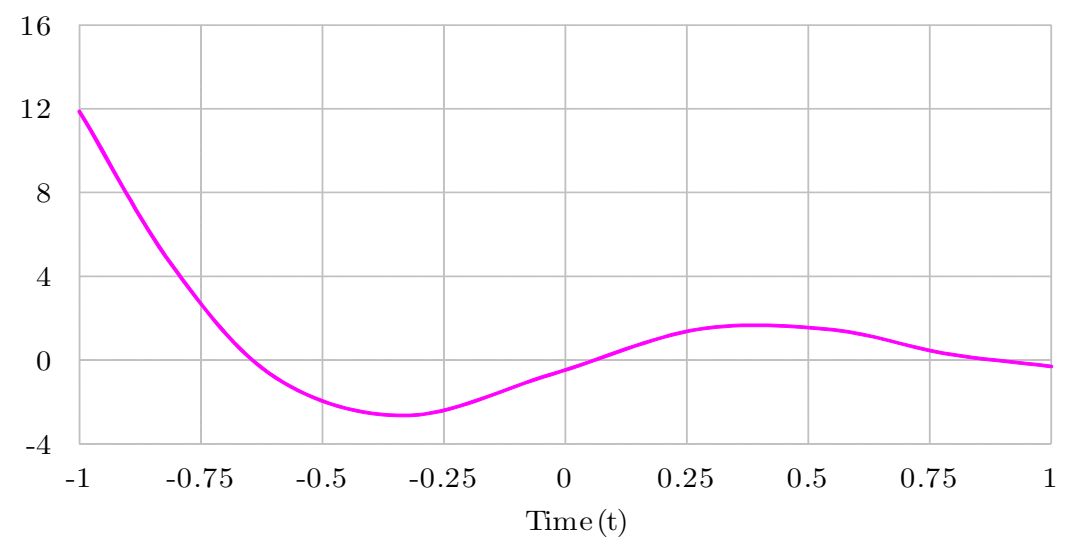

Figure 4. Control $u(t)$ of Example 2

generate the optimal solution. Figures and tables contents the results of some problems. Each of these problems is solved by converting it into a quadratic programming problem using the Jacobi polynomials. The proposed method will be generalized to solve nonlinear optimal control problem with general boundary conditions. 


\section{References}

[1] W. W. Bell, Special function for scientists and engineers, D. Van Nostrand Co., Ltd., London-Princeton, N.J.-Toronto, Ont., 1968.

[2] R. Bhattacharya, A MATLAB Toolbox for Optimal Trajectory Generation, 2006.

[3] A. H. Bhrawy and S. I. El-Soubhy, Jacobi spectral Galerkin method for the integrated forms of second-order differential equations, Applied Mathematics and Computation 217 (2010), 2684-2697.

[4] E. H. Doha, On the construction of recurrence relations for the expansion and connection coefficients in series of Jacobi polynomials, J. Phys. A 37 (2004), no. 3, 657-675.

[5] E. H. Doha and H. M. Ahmed, Efficient algorithms for construction of recurrence relations for the expansion and connection coefficients in series of quantum classical orthogonal polynomials, Journal of Advanced Research - Cairo Univ. 1, 193-207 (2010).

[6] E. H. Doha and A. H. Bhrawy, Efficient spectral-Galerkin algorithms for direct solution of fourth-order differential equations using Jacobi polynomials, Appl. Numer. Math. 58 (2008), no. 8, 1224-1244.

[7] E. H. Doha and A. M. Waleed, Efficient spectral-Galerkin algorithms for direct solution of second-order equations using ultraspherical polynomials, SIAM J. Sci. Comput. 24 (2003), no. 2, 548-571.

[8] T. M. El-Gindy and M. S. Salim, Penalty function with partial quadratic interpolation technique in the constrained optimization problems, J. Inst. Math. Comput. Sci. Math. Ser. 3 (1990), no. 1, 85-90.

[9] D. Gottlieb and S. A. Orszag, Numerical Analysis of Spectral Methods: Theory and Applications, CBMS-NSF Regional Conference Series in Applied Mathematics, No. 26. Society for Industrial and Applied Mathematics, Philadelphia, Pa., 1977.

[10] IPOPT open source NLP solver. https://projects.coin-or.org/Ipopt.

[11] H. Jadu, Spectral method for constrained linear-quadratic optimal control, Math. Comput. Simulation 58 (2002), no. 2, 159-169.

[12] W. Kang and N. Bedrossian, Pseudospectral optimal control theory makes debut flight, saves nasa $1 \mathrm{~m}$ in under three hours, SIAM News 40 (2007).

[13] W. Kang, Q. Gong, I. M. Ross, and F. Fahroo, On the Convergence of Nonlinear Optimal Control Using Pseudospectral Methods for Feedback Linearizable Systems, Internat. J. Robust Nonlinear Control 17 (2007), no. 14, 1251-1277.

[14] H. T. Rathod, B. Venkatesudu, K. V. Nagaraja, and Md. S. Islam, Gauss LegendreGauss Jacobi quadrature rules over a tetrahedral region, Appl. Math. Comput. 190 (2007), no. 1, 186-194.

[15] I. M. Ross and F. Fahroo, Pseudospectral knotting methods for solving nonsmooth optimal control problems, Journal of Guidance Control and Dynamics 27 (2004), 397-405.

[16] J. Shen and L. Wang, Some recent advances on spectral methods for unbounded domains, Commun. Comput. Phys. 5 (2009), no. 2-4, 195-241.

[17] G. Szegö, Orthogonal Polynomials, Amer. Math. Soc. Colloq. Publ., Vol. 23, 1985.

[18] M. Urabe, Numerical solution of multi-point boundary value problems in Chebyshev series. Theory of the method, Numer. Math. 9 (1967), 341-366.

[19] J. Vlassenbroeck, A Chebyshev polynomial method for optimal control with state constraints, Automatica J. IFAC 24 (1988), no. 4, 499-506.

Department of Mathematics

FACUlty OF SCIENCE

HELWAN UNIVERSITY

CAIRo, Egypt

E-mail address: m_el_kady@hotmail.com 